\title{
Mortalidad por cáncer mamario, prostático y cervicouterino, años perdidos y costos de los programas. México, 2013 a 2016
}

\author{
Carlos Navarrete-Valero y Carlos Navarrete-Vázquez
}

Instituto de Seguridad y Servicios Sociales de los Trabajadores del Estado, Estado de México, México

\begin{abstract}
Resumen
Introducción: En México se realizan fuertes erogaciones en recursos para la detección oportuna del cáncer cervicouterino y mamario, pero la Secretaría de Salud y el Instituto Mexicano del Seguro Social no cuentan con un programa para la detección de cáncer prostático. Objetivos: Comparar la mortalidad por cáncer mamario, cervicouterino y prostático en México, así como de los años perdidos de 2013 a 2016, contra los costos de los programas. Método: De la mortalidad general por residencia se tomaron las defunciones por cada tipo de cáncer con base en la CIE-10; los años perdidos fueron obtenidos conforme la esperanza de vida de México: 72 y 77 años para hombres y mujeres. Resultados: La mortalidad por cáncer prostático fue mayor a la de cáncer mamario, pero los años perdidos en mujeres por cáncer mamario fue mayor. El costo de los programas para cáncer mamario y prostático de las dos instituciones de 2013 a 2016 fue de \$3 036322156 m.n. Conclusión: Los programas de las instituciones no han impactado en beneficio de la población a pesar de su costo y no se ha dimensionado correctamente el cáncer prostático. Es necesario evaluar y rediseñar estrategias que optimicen el gasto y beneficien a la población.
\end{abstract}

PALABRAS CLAVE: Mortalidad. Cáncer mamario. Cáncer prostático. Cáncer cervicouterino. Años de vida perdidos.

\begin{abstract}
Introduction: In Mexico, large expenditure is made on resources for cervical and breast cancer early detection, but the Ministry of Health and the Mexican Institute of Social Security do not have a program for the detection of prostate cancer. Objective: To compare breast, cervical and prostate cancer mortality, as well as years lost in Mexico from 2013 to 2016 versus the cost of programs. Method: Overall mortality figures were taken from the number of deaths for each type of cancer based on ICD-10. The number of years lost were obtained according to life expectancy in Mexico (72 years for men and 77 years for women). Results: Prostate cancer mortality is higher than that of breast cancer, but lost years in women due to breast cancer are higher. The cost of programs for breast and prostate cancer from 2013 to 2016 in both institutions was 3036 322156 Mexican Pesos. Conclusion: Institutional programs have not had an impact to the benefit of the population in spite of their cost and prostate cancer has not been correctly evaluated. It is necessary for strategies to be assessed and redesigned in order to optimize expenditure and benefit the population.
\end{abstract}

KEY WORDS: Mortality. Breast cancer. Prostate cancer. Cervical cancer. Years of Life Lost.

Fecha de recepción: 30-04-2018

Fecha de aceptación: 20-08-2018

DOI: 10.24875/GMM.18004369
Gac Med Mex. 2018;154:665-670

Disponible en PubMed www.gacetamedicademexico.com 


\section{Introducción}

La Organización Mundial de la Salud menciona que entre los principales cánceres que atacan a las mujeres en el mundo se encuentran el cáncer mamario y el cervicouterino: en 2015 fallecieron en el mundo 570896 mujeres por el primero y 279311 por el segundo; en cuanto a los hombres, 1064 murieron por cáncer mamario y 343898 por cáncer prostático. ${ }^{1}$

Si se compara la frecuencia de cáncer mamario en la región de las Américas en mujeres menores de 65 años, es mayor en América Latina y el Caribe con $57 \%$, que en América del Norte con $41 \%{ }^{2}$ En cuanto al cáncer prostático se diagnostican 413000 casos nuevos cada año y fallecen 85000 hombres; ${ }^{3}$ solo en la región de América del Norte en 2015 fallecieron 35155 hombres por este cáncer y en Centroamérica $1168 .^{4}$

Sobre el cáncer cervicouterino, en la región de las Américas en 2012 más de 83000 mujeres fueron diagnosticadas de cáncer cervicouterino y casi 36000 fallecieron por esta enfermedad. Si se mantienen las tendencias actuales, el número de muertes en las Américas aumentará en $45 \%$ para 2030. Las tasas de mortalidad son tres veces más altas en América Latina y el Caribe que en Norteamérica, lo que evidencia enormes desigualdades en salud. ${ }^{5}$

Los Estados Unidos Mexicanos, con base en el Censo de Población y Vivienda de 2010, efectuado por el Instituto Nacional de Estadística y Geografía, se encontraba constituido por 112336538 habitantes, ${ }^{6}$ para la Encuesta Intercensal efectuada en el año 2015 la población total fue de $119530753^{7}$ y las proyecciones de población del Consejo de Población y Vivienda menciona para 2016 una población nacional de 122273 473, 59644308 hombres y 62629165 mujeres, $^{8}$ por lo que la diferencia entre hombres y mujeres es de poco más de dos millones.

La Constitución Política de Estados Unidos Mexicanos, en su artículo 4 indica que toda persona tiene derecho a la protección de la salud ${ }^{9}$ y la Encuesta Intercensal 2015 efectuada por el Instituto Nacional de Estadística y Geografía en el rubro de servicios de salud reporta que $17 \%$ de la población nacional no se encuentra afiliada a instituciones de salud y que la población afiliada lo está a una de tres instituciones: $49.90 \%$ a la Secretaría de Salud, con el Seguro Popular, $39.18 \%$ al Instituto Mexicano del Seguro Social y $7.71 \%$ al Instituto de Seguridad y
Servicios Sociales de los Trabajadores del Estado. Dado que las tres instituciones abarcan a $96.79 \%$ de la población afiliada, es importante que cuenten con programas de detección temprana de cáncer mamario en mujeres y hombres, así como para cervicouterino y prostático, cuyo desarrollo requiere fuertes erogaciones de recursos. A pesar de ello, la Secretaría de Salud y el Instituto Mexicano del Seguro Social no cuentan con un programa para la detección de cáncer prostático y se da prioridad a la detección oportuna de cáncer mamario y cervicouterino en mujeres. ${ }^{10-12}$

Sobre la esperanza de vida en la región de las Américas, la Organización Mundial de la Salud reportó para 2015 una esperanza de vida para los hombres de 74 años y para las mujeres de 79.9 años. ${ }^{13}$ En cuanto a México, el Instituto Nacional de Estadística y Geografía ${ }^{14}$ reportó para 2016 una esperanza de vida de 72.6 años para los hombres y de 77.8 para las mujeres, por debajo de la esperanza de vida de la región.

Tomando como referencia los datos de mortalidad de la Organización Mundial de la Salud y de la Organización Panamericana de la Salud sobre el cáncer mamario, cervicouterino y prostático, así como la edad promedio de esperanza de vida en la región y que un diagnóstico temprano eficaz puede facilitar la detección del cáncer en una fase más precoz, lo que posibilita la aplicación de tratamientos que suelen ser más eficaces, menos complejos y menos costosos, ${ }^{15}$ se debe determinar si los programas de las instituciones de salud de México están impactando favorablemente en la población y no solo conocer el número de actividades para la detección precoz. Aunado a esto, mucho se habla de la prioridad a la mujer por la mortalidad por cáncer mamario, pero ¿qué sucede con el cáncer prostático? Debido a lo anterior, el objetivo de este trabajo fue conocer lo siguiente para el periodo 2013 a 2016:

1. Si en México han disminuido las defunciones por cáncer mamario en mujeres y en hombres, así como las defunciones por cáncer cervicouterino y cáncer prostático.

2. Si en el país la mortalidad acumulada de mujeres por cáncer mamario es mayor a la mortalidad de los hombres por cáncer prostático.

3. La mortalidad por entidad federativa acumulada por cáncer mamario en mujeres, cáncer prostático y cáncer cervicouterino.

4. Los años perdidos acumulados en números absolutos por defunciones debidas a cáncer 
mamario en mujeres y hombres, así como a cáncer cervicouterino y cáncer prostático, en relación con la esperanza de vida para mujeres y hombres mexicanos.

5. Si en México han disminuido los años perdidos por defunciones debidas a cáncer mamario en mujeres y hombres, así como por defunciones por cáncer cervicouterino y cáncer prostático.

6. El costo de los programas de prevención de cáncer mamario, cervicouterino y prostático, en la Secretaría de Salud, el Instituto Mexicano del Seguro Social e Instituto de Seguridad y Servicios Sociales de los Trabajadores del Estado.

\section{Método}

El universo de estudio fue la población de los Estados Unidos Mexicanos y la muestra el $100 \%$ de los fallecimientos por cáncer mamario en mujeres y hombres, así como por cáncer prostático y cervicouterino, de 2013 a 2016. Los fallecimientos se tomaron de las defunciones generales por residencia reportadas por el Instituto Nacional de Estadística y Geografía registradas en ese periodo.

Para conocer el número de muertes por cáncer mamario en mujeres y hombres por edad, de la Décima Revisión de la Clasificación Internacional de Enfermedades ${ }^{16}$ se tomaron siguiente claves:

- C500 tumor maligno del pezón y areola mamaria.

- C50 tumor maligno de la porción central de la mama.

- C502 tumor maligno del cuadrante superior interno de la mama.

- C503 tumor maligno del cuadrante inferior interno de la mama.

- C504 tumor maligno del cuadrante superior externo de la mama.

- C505 tumor maligno del cuadrante inferior externo de la mama.

- C506 tumor maligno de la prolongación axilar de la mama.

- C508 lesión de sitios contiguos de la mama.

- C509 tumor maligno de la mama, parte no especificada.

Para el número de muertes por cáncer de próstata por edad:

- C61 tumor maligno de la próstata.

En cuanto al número de muertes por cáncer cervicouterino por edad:
- C53 tumor maligno del cuello del útero.

- C53.0 tumor maligno del endocérvix.

- C53.1 tumor maligno del exocérvix.

- C53.8, lesión de sitios contiguos del cuello del útero.

- C53.9 tumor maligno del cuello del útero, sin otra especificación.

- C54 tumor maligno del cuerpo del útero.

- C54.0 tumor maligno del istmo uterino.

- C54.1 tumor maligno del endometrio.

- C54.2 tumor maligno del miometrio.

- C54.3 tumor maligno del fondo del útero.

- C54.8 lesión de sitios contiguos del cuerpo del útero.

- C54.9 tumor maligno del cuerpo del útero, parte no especificada.

- C55 tumor maligno del útero, parte no especificada.

Para determinar los años perdidos se tomó como base para las mujeres la esperanza de vida de 77 años y para los hombres de 72 años, por lo cual para cada edad de fallecimiento se restó a los hombres 72 años y a las mujeres 77 años, el resultado fueron los años perdidos por fallecimiento, que se multiplicaron por el número de defunciones en cada edad, para tener el número absoluto de los años perdidos por edad.

En cuanto al costo de los programas por institución de 2013 a 2016 se solicitó a cada institución la información mediante la Plataforma Nacional de Transparencia Gobierno Federal. Los datos fueron procesados en Excel para tabular los resultados. A cada valor de edad por año se le asoció el número de fallecimientos que fueron registrados; para el estudio se utilizó la frecuencia absoluta y la frecuencia absoluta acumulada ya que esta fue la suma de las variables de 2013 a 2016, para posteriormente representarlos en tablas.

\section{Resultados}

En la Tabla 1 se exponen las defunciones por tipo de cáncer y cada año de 2013 a 2016, en este último todos los fallecimientos son al alza en comparación con 2015, aunado a esto se observa que los fallecimientos de forma acumulada de 2013 a 2016 por cáncer prostático representan la mayor cantidad con 25 685, en comparación con los demás tipos de cáncer.

En la Figura 1 se muestra la distribución de la mortalidad por entidad federativa: el Estado de México, la Ciudad de México, Veracruz y Jalisco ocuparon los 
Tabla 1. Defunciones en los Estados Unidos Mexicanos por tipo de cáncer y sexo. 2013-2016

\begin{tabular}{lccccc}
\hline Defunciones & $\begin{array}{c}\mathbf{2 0 1 3} \\
\text { (n) }\end{array}$ & $\begin{array}{c}\mathbf{2 0 1 4} \\
\text { (n) }\end{array}$ & $\begin{array}{c}\mathbf{2 0 1 5} \\
\text { (n) }\end{array}$ & $\begin{array}{c}\mathbf{2 0 1 6} \\
\text { (n) }\end{array}$ & $\begin{array}{c}\mathbf{2 0 1 3 - 2 0 1 6} \\
\text { acumulado }\end{array}$ \\
\hline $\begin{array}{l}\text { De mujeres por cáncer } \\
\text { mamario }\end{array}$ & 5548 & 5997 & 6273 & 6650 & 24468 \\
$\begin{array}{l}\text { De hombres por cáncer } \\
\text { mamario }\end{array}$ & 49 & 54 & 31 & 43 & 177 \\
$\begin{array}{l}\text { Total por cáncer } \\
\text { mamario }\end{array}$ & 5597 & 6051 & 6304 & 6693 & 24645 \\
$\begin{array}{l}\text { Por cáncer } \\
\text { cervicouterino }\end{array}$ & 4748 & 5059 & 5046 & 5144 & 19997 \\
Por cáncer prostático & 6023 & 6296 & 6447 & 6919 & 25685 \\
\hline
\end{tabular}

Tabla 2. Total de años perdidos por defunciones antes de la edad denominada esperanza de vida. 2013-2016

\begin{tabular}{lccccc}
\hline Años perdidos & $\mathbf{2 0 1 3}$ & $\mathbf{2 0 1 4}$ & $\mathbf{2 0 1 5}$ & $\mathbf{2 0 1 6}$ & $\begin{array}{r}\mathbf{2 0 1 3 - 2 0 1 6} \\
\text { acumulado }\end{array}$ \\
\hline $\begin{array}{l}\text { De mujeres por } \\
\text { cáncer mamario }\end{array}$ & 103475 & 112916 & 115426 & 122023 & 453840 \\
$\begin{array}{l}\text { De hombres por } \\
\text { cáncer mamario }\end{array}$ & 356 & 511 & 318 & 468 & 1653 \\
$\begin{array}{l}\text { Por cáncer } \\
\text { cervicouterino }\end{array}$ & 86421 & 92341 & 92057 & 94858 & 365677 \\
$\begin{array}{l}\text { Por cáncer } \\
\text { prostático }\end{array}$ & 11864 & 13415 & 13168 & 13646 & 52093 \\
\hline
\end{tabular}

primeros lugares en mortalidad por los tipos de cáncer estudiados.

En la Tabla 2 se muestran los años perdidos por la mortalidad debida a estos tipos de cáncer de 2013 a 2016: fueron mayores en 2016 en comparación con 2015. Sobre los años perdidos de forma acumulada, las mujeres ostentaron la mayor cantidad de años perdidos por cáncer mamario con 453840 , seguidas por los hombres con 52093 años perdidos debido a cáncer prostático.

Sobre el costo de los programas, en la Tabla 3 se puede observar que la Secretaría de Salud redujo su gasto en 2014 y 2015 en relación con 2013, a diferencia del Instituto Mexicano del Seguro Social que lo ha incrementado paulatinamente. Entre las dos instituciones, entre 2013 y 2016 erogaron \$2 752321069 de pesos; el Instituto de Seguridad y Servicios Sociales de los Trabajadores del Estado no proporcionó su información debido a que no llevaba la cuantificación del costo de los programas.

\section{Conclusión}

Se determinó que son mayores los fallecimientos de hombres por cáncer prostático que de mujeres por cáncer mamario, a diferencia de los años perdidos, mayores en las mujeres, lo que traduce que los hombres fallecen más cerca a la edad denominada esperanza de vida y que las mujeres mueren en edades más tempranas a la esperanza de vida.

Existen otros métodos para calcular los años perdidos cuando se dispone del número de defunciones por grupos de edad, asumiendo una distribución uniforme de defunciones en cada grupo. También se pueden expresar como tasa, tanto si se dispone de los datos individualizados como agrupados, sin embargo, al igual que las tasas brutas de mortalidad, las tasas de años de vida perdidos pueden estar influidas por variables confusoras, como generalmente ocurre con la estructura de edad de la población. De igual forma, se puede calcular con la diferencia entre la edad de muerte de cada fallecido de una población y un número concreto de años que ha de fijarse, luego se suman los años perdidos por parte de cada individuo que fallece antes del umbral fijado por una determinada causa en una población; la suma dará el total de años perdidos por dicha causa en esa población. ${ }^{17}$ Se eligió esta forma de cálculo para evitar variables confusoras y debido a que los años de vida perdidos sirven para determinar la mortalidad prematura.

La mayor mortalidad por entidad federativa fue para la Ciudad de México, seguida por el Estado de México, Jalisco y Veracruz. Se podría pensar que sería proporcional a la cantidad de población que ostentan, sin embargo, al observar la población total de la Encuesta Intercensal del Instituto de Geografía y Estadística 2015, el primer lugar fue para el Estado de México con 16187608 habitantes, seguido por la Ciudad de México con 8918 653, Veracruz con 8112505 y Jalisco con 7844830 , por lo que se puede inferir que no es proporcional. De ahí la importancia de efectuar un estudio para tener las tasas de mortalidad por 100000 habitantes por entidad federativa, para precisar mejor las diferencias entre las entidades.

Los programas de detección oportuna no son eficaces aun cuando en México se ha dado prioridad a la detección temprana del cáncer mamario y cervicouterino, ya que existe una normatividad para uno y otro.

En México, no se le ha dado la importancia debida al cáncer prostático a pesar de la alta mortalidad que ocasiona. Fue hasta el 15 de diciembre de 2017 


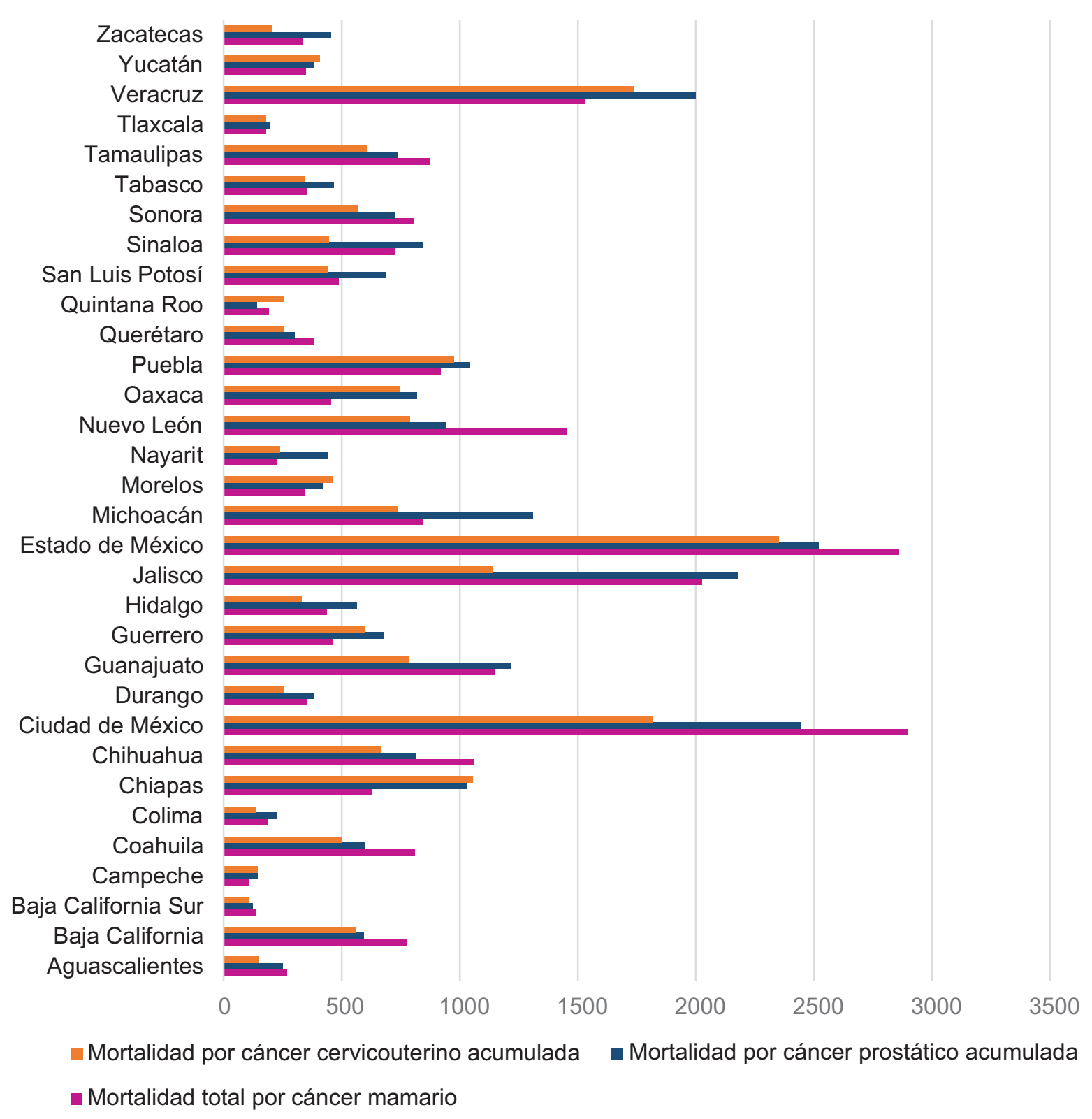

Figura 1. Mortalidad por entidad federativa acumulada por tipo de cáncer, 2013-2016.

Tabla 3. Costo de los programas de las principales instituciones de salud de México

\begin{tabular}{|c|c|c|c|c|c|c|}
\hline Institución & Programa & 2013 & 2014 & 2015 & 2016 & $\begin{array}{l}\text { 2013-2016 } \\
\text { acumulado }\end{array}$ \\
\hline Secretaría de Salud & Cáncer mamario y cervicouterino & $\$ 507797297$ & $\$ 327024435$ & $\$ 467583885$ & $\$ 579714318$ & $\$ 1882119934$ \\
\hline $\begin{array}{l}\text { Instituto Mexicano del } \\
\text { Seguro Social }\end{array}$ & Cáncer mamario y cervicouterino & $\$ 178927042$ & $\$ 191159455$ & $\$ 223692645$ & $\$ 276422003$ & $\$ 870201135$ \\
\hline
\end{tabular}

cuando se publicó la Norma oficial mexicana NOM048-SSA2-2017, para la prevención, detección, diagnóstico, tratamiento, vigilancia epidemiológica y promoción de la salud sobre el crecimiento prostático benigno (hiperplasia de la próstata) y cáncer de próstata (tumor maligno de la próstata). Por esto, en el periodo del estudio la Secretaría de Salud y el Instituto Mexicano del Seguro Social reportaron no tener un programa para el cáncer prostático, a diferencia del cáncer mamario, cuyo programa iniciaron en 2001 
y 2002; el del cáncer cervicouterino lo comenzaron en 1973 y 2001, respectivamente. El costo de ambos programas por las dos instituciones de forma acumulada de 2013 a 2016 fue de casi 3 mil millones de pesos. El Instituto de Seguridad y Servicios Sociales de los Trabajadores del Estado dice tener un programa para cada uno de los tres tipos de cáncer, sin embargo, no lleva la cuantificación del costo de estos, por lo cual no proporcionó la información.

Por lo anterior, las instituciones deben replantear sus programas para modificar la tendencia y que consideren las nuevas directrices sugeridas por la Organización Mundial de la Salud, como las prácticas esenciales para el control integral del cáncer cervicouterino, ya que uno de los objetivos de la salud pública es reducir la incidencia, la prevalencia y el número de defunciones causadas por cáncer. ${ }^{18}$

Se debe tener una visión integral en la elaboración de los programas, por ejemplo, considerar en el cáncer cervicouterino los tres componentes interdependientes mencionados por la Organización Mundial de la Salud; la prevención primaria, secundaria y terciaria con las intervenciones previstas para cada componente, en especial para reducir los años perdidos por muerte antes de la esperanza de vida, particularmente en las mujeres con cáncer mamario, el cual ocupa el primer lugar en años perdidos.

Una variable que las instituciones de salud de México no han considerado es el cáncer mamario en hombres, aun cuando es muy infrecuente. Deben realizarse programas para difundir información al sexo masculino, en particular sobre la autoexploración para una detección temprana.

Si bien es cierto que $17 \%$ de la población mexicana no tiene acceso a los servicios de salud, también es cierto que entre la Secretaría de Salud, el Instituto Mexicano del Seguro Social y el Instituto de Seguridad y Servicios Sociales de los Trabajadores del Estado atiende aproximadamente a $96 \%$ de la población afiliada, por lo que sus programas y presupuesto tendrían que impactar en la disminución de los años perdidos por mortalidad debida a cáncer mamario, cervicouterino y prostático.

Es necesario efectuar estudios por cada entidad federativa del país, con la finalidad de particularizar las edades en la que se debe realizar el cribaje y hacer detecciones tempranas para llevar a cabo tratamientos más oportunos, que reducirán los años perdidos.

\section{Bibliografía}

1. Organización Mundial de la Salud. [Sitio web]. Estimaciones de salud global 2015 muertes estimadas por edad, sexo y causa. Disponible en: http://www. who.int/healthinfo/global/_burden_disease/estimates/en/index1.html

2. Organización Panamericana de la Salud/Organización Mundial de la Salud. [Sitio web]. Cáncer de mama. Disponible en: http://www.paho.org/ hq/index.php? option=com_content\&view=article\&id=5041\&Itemi$\mathrm{d}=3639$ \&lang $=$ es\&ltemid $=3639$

3. Organización Panamericana de la Salud/Organización Mundial de la Salud. [Sitio web]. Expertos regionales discuten enfoques para el tamizaje y detección temprana del cáncer de próstata en las Américas. Disponible en: http://www.paho.org/hq/index.php?option=com_content\&view=article\&id=13818\&ltemid $=42459$ \&lang $=e s$

4. Organización Panamericana de la Salud/Organización Mundial de la Salud. [Sitio web]. Datos de mortalidad. Disponible en: https://hiss.paho. org/pahosys/idc.php

5. Organización Panamericana de la Salud/Organización Mundial de la Salud. [Sitio web]. Cómo se desarrolla el cáncer cervicouterino. Disponible en: http://www.paho.org/hq/index.php?option=com_content\&view=article\&i$d=5521$ \&ltemid=3962\&lang=es

6. Instituto Nacional de Estadística y Geografía. [Sitio web]. Censo de Población y Vivienda 2010. Cuestionario básico. Disponible en: http://www3. inegi.org. $\mathrm{mx} / \mathrm{sistemas} /$ tabuladosbasicos/default.aspx?c=27302\&s=est

7. Instituto Nacional de Estadística y Geografía. [Sitio web]. Encuesta intercensal INEGI 2015. México: INEGI; 2015. Disponible en: http://www. beta.inegi.org.mx/proyectos/enchogares/especiales/intercensal/

8. Consejo Nacional de Población. [Sitio web]. Proyecciones de la población de México 2010-2050 Indicadores demográficos. Disponible en: http://www.conapo.gob.mx/es/CONAPO/Proyecciones

9. Constitución Política de los Estados Unidos Mexicanos. Disponible en: http://www.diputados.gob.mx/LeyesBiblio/pdf/1.pdf

10. Instituto Nacional de Transparencia, Acceso a la Información y Protección de Datos Personales. [Sitio web]. Información pública folio 0001200015818. Disponible en: https://www.infomex.org.mx/gobiernofederal/moduloPublico/moduloPublico.action

11. Instituto Nacional de Transparencia, Acceso a la Información y Protección de Datos Personales. [Sitio web]. Información pública folio 0064100167018. Disponible en: https://www.infomex.org.mx/gobiernofederal/moduloPublico/moduloPublico.action

12. Instituto Nacional de Transparencia, Acceso a la Información y Protección de Datos Personales. [Sitio web]. Información pública folio 0063700026718. Disponible en: https://www.infomex.org.mx/gobiernofederal/moduloPublico/moduloPublico.action

13. World Health Organization. [Sitio web]. Life expectancy data by WHO region. Disponible en: http://apps.who.int/gho/data/view.main.SDG2016LEXREGv?lang=en

14. Instituto Nacional de Estadística y Geografía. [Sitio web]. Esperanza de vida. Disponible en: http://cuentame.inegi.org.mx/poblacion/esperanza. aspx?tema $=\mathrm{P}$

15. Organización Mundial de la Salud. [Sitio web]. El diagnóstico temprano del cáncer salva vidas y reduce los costos de tratamiento. Disponible en: http:// www.who.int/mediacentre/news/releases/2017/early-cancer-costs/es/

16. Organización Panamericana de la Salud/Organización Mundial de la salud. [Sitio web]. ICD 10 en línea, Volumen 1, Español. Disponible en: http://ais.paho.org/classifications/Chapters/

17. Organización Panamericana de la Salud/Organización Mundial de la salud. [Sitio web]. Demografía. Disponible en: https://www.paho.org/nic/index.php?option=com_docman\&view=download\&alias=45-bases-conceptuales-demograficas\&category_slug=publicaciones-anteriores\&ltemid=235

18. Organización Panamericana de la Salud/Organización Mundial de la salud. Control Integral del cáncer cervicouterino, guía de prácticas esenciales 2016. Segunda edición. Washington, DC: OPS; 2016. 